\title{
Blue Phosphorene Monolayers as Potential Nano Sensors for Volatile Organic Compounds Under Point Defects
}

\author{
Suyang Sun, ${ }^{1}$ Tanveer Hussain, ${ }^{2 *}$ Wei Zhang, ${ }^{1}$ and Amir Karton ${ }^{2}$
}

${ }^{1}$ Center for Advancing Materials Performance from the Nanoscale, State Key Laboratory for Mechanical Behavior of Materials, Xi' an Jiaotong University, Xi' an 710049, P.R. China.

${ }^{2}$ School of Molecular Sciences, University of Western Australia, Perth, WA 6009, Australia.

\begin{abstract}
Based on spin-polarized DFT calculations, we have studied the interaction mechanism of recently synthesized blue phosphorene (BlueP) monolayers towards selected key volatile organic compounds (VOCs) such as acetone, ethanol and propanal. Our binding energy analysis shows that pristine BlueP weakly binds the VOCs and that this binding does not appreciably change the electronic properties of the monolayer - a prerequisite for any sensing material. However, mono, di, and tri-vacancy defects and $\mathrm{Si} / \mathrm{S}-$ substitutional doping significantly enhance the binding energies with VOCs. Density of state (DOS) calculations show that upon adsorption of VOCs, mono-vacancy and Ssubstituted BlueP monolayers undergo a major change in electronic structure, which make them potential candidates for VOCs sensing materials. By contrast, binding of VOCs to di- and tri-vacancy and Si-substitution sites does alter the electronic structure of BlueP monolayers drastically, therefore, are not qualified for VOCs sensing applications.
\end{abstract}

\section{Cite as:}

S. Sun, T. Hussain, W. Zhang, A. Karton, Applied Surface Science, 486, 52-57 (2019). https://doi.org/10.1016/j.apsusc.2019.04.223

Keywords: DFT, Gas sensing, 2D materials, Substitution defects, Vacancy defects

*Corresponding author. Email: tanveer.hussain@uwa.edu.au 


\section{Introduction}

The detection of volatile organic compounds (VOCs) has become an important task, because high concentrations of VOCs in indoor air may be harmful to human health ${ }^{1-5}$. VOCs are organic compounds that have low boiling points and thus high vapor pressures at room temperature. A wide range of commonly used materials may release significant amounts of VOCs into indoor air ${ }^{5}$, some of which are identified to be toxic and carcinogenic even at sub-ppb concentrations ${ }^{1,6}$. Therefore, it is important to design efficient sensors capable of detecting and capturing VOCs.

Semiconductor gas-sensors based on metal oxides are widely used to detect VOCs by monitoring the change in their electronic conductivity before and after the adsorption of gas molecules ${ }^{6-11}$. However, the conventional semiconductor sensors have limited resolution at the ppm level because of size and shape effects ${ }^{10}$. In addition, another fundamental reason limiting the resolution of such devices is the intrinsic noise due to the thermal motion of charges and defects, which may exceed the signal of the target molecules ${ }^{12}$.

One approach for improving the resolution of semiconductor sensors is utilizing nanotechnologies. Metal oxide nanostructures offer a larger surface-to-volume ratio, i.e. more adsorption sites for the target gas molecules, and consequently higher sensitivity than conventional materials. For example, Pt-decorated $\operatorname{In}_{2} \mathrm{O}_{3}$ nanoparticles can reach up to ppb resolution ${ }^{6,13}$. Another way to improve the resolution of semiconductor gas sensors is to employ emerging two-dimensional (2D) materials with attractive properties such as high carrier mobility and large surface-to-volume ratios, which make them ideal materials for many applications in addition to gas sensing ${ }^{1421}$. Several recent works have revealed that sensing devices based on graphene ${ }^{12,22,23}$ or transition metal dichalcogenides (TMDs) like $\mathrm{MoS}_{2}{ }^{24,25}$ can efficiently sense various gas molecules.

A number of recent theoretical and experimental studies have demonstrated the application of black phosphorene - the most stable allotrope of phosphorus - for gas sensing ${ }^{26-35}$. More recently, a new 2D phase of phosphorus with in-plane hexagonal structure called blue phosphorene (BlueP) has been designed theoretically ${ }^{36}$ and 
synthesized experimentally ${ }^{37}$. BlueP has been found to be as stable as black phosphorene $^{31}$, and its narrow band gap makes it suitable for gas-sensing applications.

In this work, we employ density functional theory (DFT) calculations to predict the structural, electronic and VOCs sensing properties of BlueP monolayer upon defect engineering. Various types of point defects, i.e. mono-vacancy (MV-), di-vacancy (DV-), tri-vacancy (TV-), Si- and S-substitution, are considered, because they are known to affect the binding of molecules of 2D materials by making them electrondeficient or electron-rich. Indeed, all these defective BlueP monolayers show an improvement in the binding of VOCs (acetone, ethanol and propanal in this work) with respect to the pristine one, and major changes in electronic structure are observed for MV- and S-substituted BlueP monolayers, making them potential candidates for VOCs sensing.

\section{Computational details}

DFT calculations were performed using the VASP $\operatorname{code}^{38}$ with the projectoraugmented wave (PAW) pseudopotentials ${ }^{39}$ and Perdew-Burke-Ernzerhof (PBE) gradient-corrected functionals ${ }^{40}$. Van der Waals interactions were treated by using the Grimme's method ${ }^{41}$. A cutoff energy of $600 \mathrm{eV}$ for plane waves was used. Freestanding BlueP monolayer was modeled by a $5 \times 5 \times 1$ hexagonal supercell consisting of 50 phosphorus atoms with a vacuum spacing of $25 \AA$ along the z-direction to decouple the interaction between periodic replicas. The optimized lattice constant ahex of BlueP monolayer is $\sim 3.277 \AA$. A $4 \times 4 \times 1$ k-point mesh was used to sample the Brillouin zone. The electronic structure calculations are crosschecked by using the Heyd-ScuseriaErnzerhof (HSE06) hybrid functionals ${ }^{42}$, which in general enlarge the size of band gaps but do not lead to any qualitative difference with respect to GGA calculations. The data presented in the main text are all produced by GGA functionals, and some HSE calculations are shown in Figure S1-S2. 


\section{Results and discussion}

First of all, we perform DFT calculations to access the structural and electronic properties of pristine BlueP monolayer. The optimized structure of BlueP supercell is shown in Figure 1 (a), where $\mathrm{P}$ atoms are arranged in a planar hexagonal crystal lattice with two $\mathrm{P}$ atoms on one lattice point, similar to $\mathrm{C}$ atoms in graphene. The difference of BlueP from graphene is that the adjacent $\mathrm{P}$ atoms are not arranged in the same 2D plane, but instead they form an angle of $92.92^{\circ}$, not $120^{\circ}$. In other words, the monolayer is buckled, see the side view in Figure 1 (a). The bond length is calculated to be $\sim 2.26$ $\AA$, which agrees well with the experimental data $^{32}$.

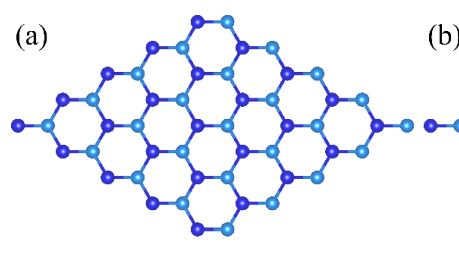

(b)

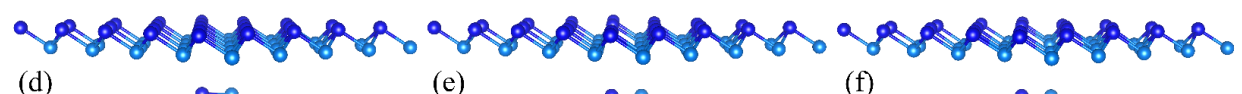

(d)

(e)
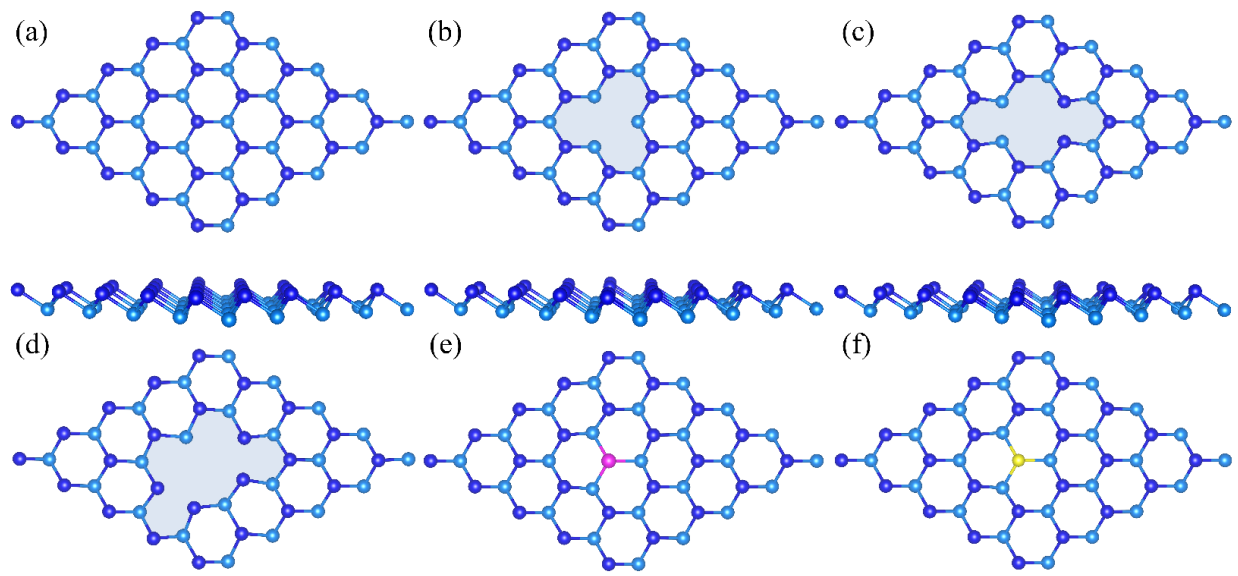

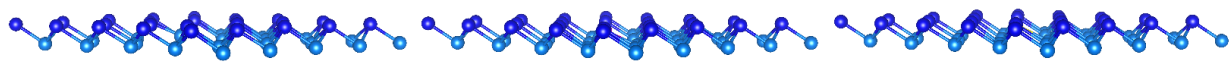

Figure 1. Optimized structures (top and side views) of blue phosphorene monolayers: (a) Pristine (BlueP), (b) monovacancy (MV-BlueP), (c) divacancy (DV-BlueP), (d) trivacancy (TV-BlueP), (e) Si doped (Si-BlueP), and (f) S doped (S-BlueP). Atomic color scheme: P atoms in upper layer of BlueP, dark blue; P atoms in lower layer of BlueP, light blue; Si, purple; and S, yellow.

To utilize BlueP as promising gas sensor for VOCs, two factors are important, a) a favorable binding of VOCs, and b) a major change in electronic structure before and after adsorption. The adsorption energies (Eads) for gas molecules (acetone, ethanol, and propanal in this work) are calculated by 


$$
E_{a d s}=E_{\text {BlueP }+ \text { gas }}-\left(E_{\text {BlueP }}+E_{\text {gas }}\right)
$$

where, the first, second and third terms on the right-hand side refer to the total energy of the BlueP monolayer adsorbed with the gas molecules, bare BlueP, and the isolated VOC molecule, respectively. For each gas molecule, several configurations with different molecular orientations and adsorption sites (Figure S3) are considered, and the configuration with the lowest energy (Table S1) is taken for further analysis (Figure 2). The adsorption energies of BlueP monolayer for acetone, ethanol, and propanal are calculated be favorable, $-0.25,-0.20$ and $-0.26 \mathrm{eV}$, respectively. However, the electronic structure of BlueP monolayers barely changes before and after the adsorption of these molecules, see the electronic density of states (DOS) in Figure 2 (d-f). This behavior makes gas sensing using pristine BlueP monolayers difficult.

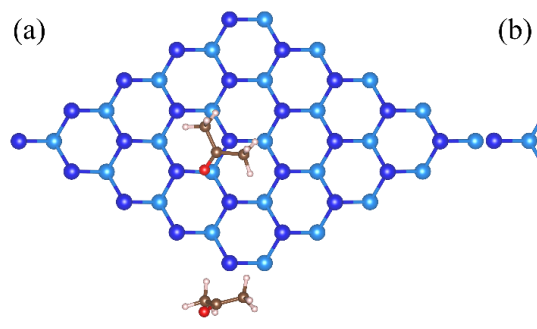

(b)

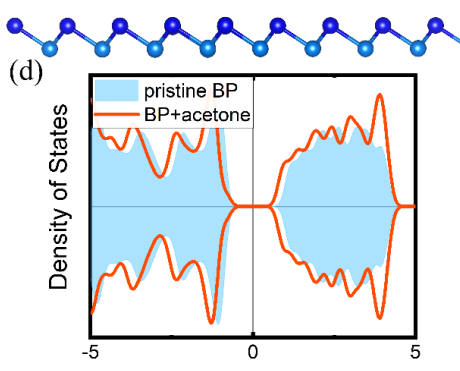

$E-E_{f}(e V)$

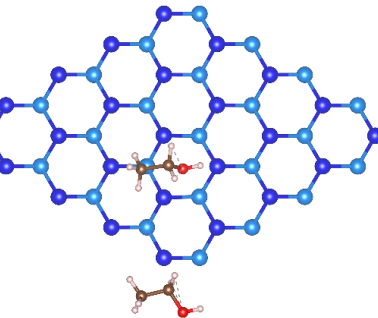

(c)
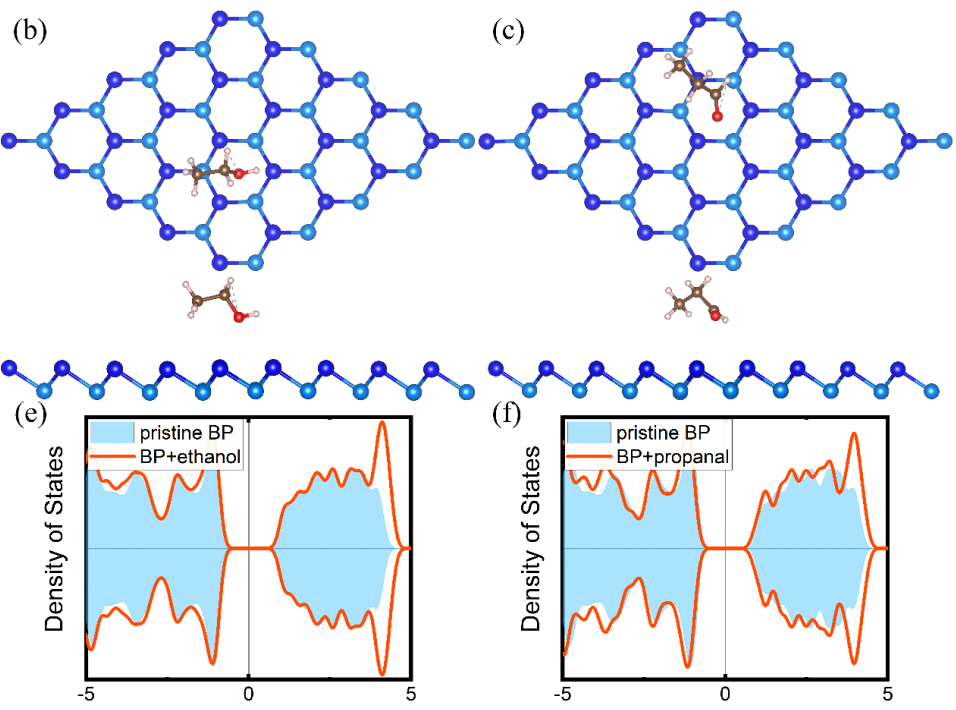

$E-E_{f}(e V)$

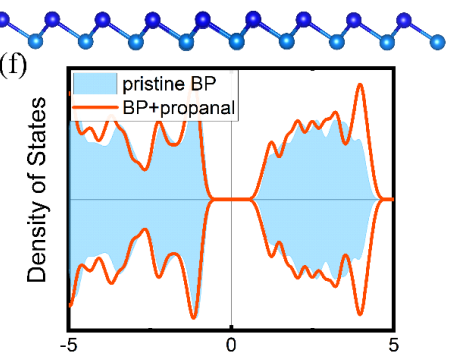

$\mathrm{E}-\mathrm{E}_{f}(\mathrm{eV})$

Figure 2. Top and side views of the energetically most stable configurations of acetone (a), ethanol (b), and propanal (c) on BlueP, and their respective density of states (DOS) plots (e-f). Atomic color scheme: $\mathrm{P}$ atoms in upper layer of BlueP, dark blue; $\mathrm{P}$ atoms in lower layer of BlueP, light blue; $\mathrm{H}$, pink; $\mathrm{C}$, brown; and $\mathrm{O}$, red.

Defect engineering using point defects, such as atomic vacancy and elemental substitution, is known as an effective route to alter the adsorption properties of 2D materials. ${ }^{27}$ Here, we consider MV-, DV-, TV-, Si-, and S-BlueP monolayers (Figure 1 
(b-f)) for adsorption of the acetone, ethanol, and propanal molecule. The adsorption energies are presented in Figure 3 (a). Clearly, all the considered point defects improve the binding of the acetone, ethanol, and propanal molecule, while the defect formation energies of DV- and TV-BlueP (Figure 3 (b)) are too high so that these two defective BlueP monolayers are more difficult for synthesis. Formation energies $\left(\mathrm{E}_{f}\right)$ in case of vacancy defects and elemental substitutions in BlueP has been calculated by the following equations (ii) and (iii), respectively

$$
\begin{array}{lr}
E_{f}=\left(E_{\text {defected }}+n E_{P}\right)-E_{\text {pristine }} & \text { ii } \\
E_{f}=E_{A-B l u e P}-\left[E_{A}+E_{(A-B l u e P)-A}\right] & \text { iii }
\end{array}
$$

Here $E_{f}$ represents the formation energy; $E_{\text {defected }}$ denotes the total energies of MVBP, DV-BP, and TV-BP, for which $n=1,2$, and 3, respectively; $E_{P}$ represents the energy of an isolated $\mathrm{P}$ atom; and $E_{\text {pristine }}$ is the total energy of a pristine BlueP monolayer. Where $A=\mathrm{Si}$ or $\mathrm{S} ; E_{A}$ denotes the energy of an isolated $A$ atom; $E_{(A-B l u e P)-A}$ represents the energy of the residue of A-BP with $A$ atom removed.

(a)

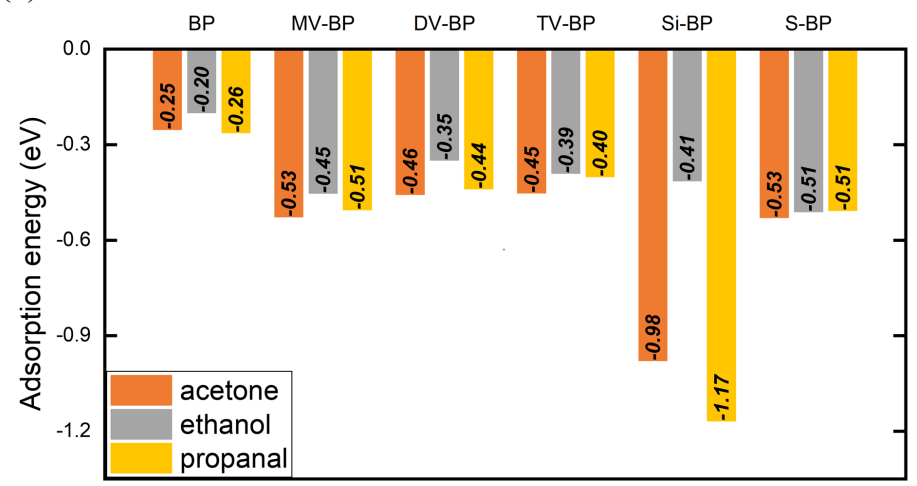

(b)

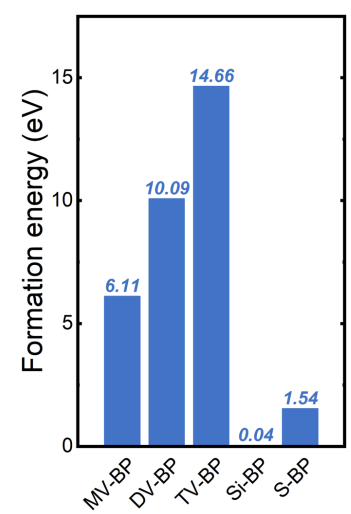

Figure 3. (a) Adsorption energies of acetone, ethanol, and propanal on different BlueP monolayers; (b) Formation energies of defective BlueP sheets. 


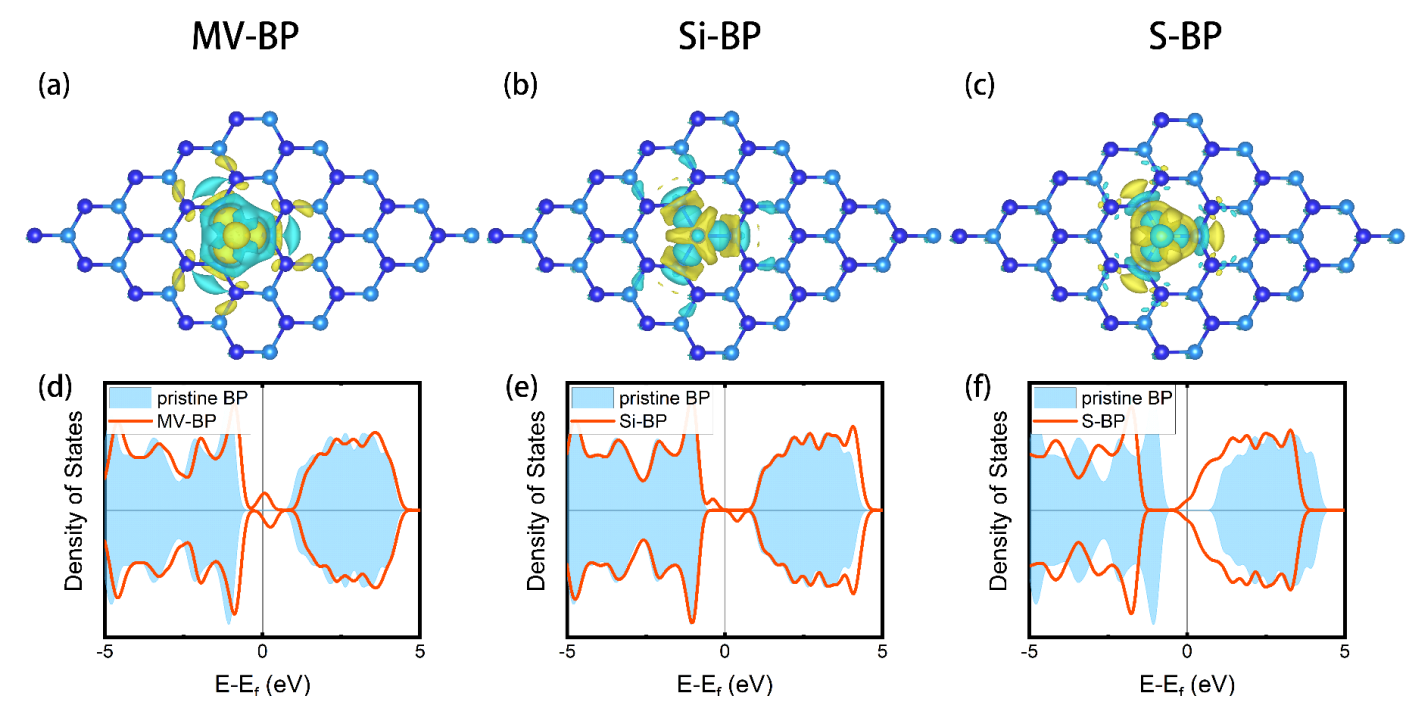

Figure 4. Isosurface charge density of (a) MV-BP, (b) Si-BP, and (c) S-BP. Yellow and cyan surfaces represent the accumulation and depletion of charge, respectively, with isovalue of 0.01 e/Å3. (d)-(f): DOS of MV-BP, Si-BP, and S-BP (red line) along with pristine BlueP (cyan filling).

\begin{tabular}{ccccc}
\hline & BP & MV-BP & Si-BP & S-BP \\
\hline Acetone & 3.02 & 1.57 & 1.26 & 2.73 \\
Ethanol & 2.69 & 1.85 & 2.02 & 2.54 \\
Propanal & 2.94 & 1.56 & 1.14 & 2.88 \\
\hline
\end{tabular}

Table 1: Equilibrium bond distances (in $\AA$ ) between VOC molecules and BlueP monolayers after relaxations along z-axis.

In the following, we investigate MV-, Si- and S-BlueP monolayer in more detail. First, we investigate the change in electronic structure upon creation of point defects without any molecule absorption. We calculate the charge density difference (CDD), spin-polarized DOS and band structure of the BlueP monolayers with and without point defects. The CDD plots presented in Figure 4 (a-c) clearly confirm a major change in charge distribution near the defect sites in the defective BlueP monolayers. In addition, 
MV and Si defects also create a couple of defect states within the band gap, while S defect moves the Fermi level to the tail of the conduction band (Figure 4 (d-f) and Figure S4). These changes in electronic structure of defective BlueP monolayers should give rise to the change in their absorption properties.

Next, we study the changes in geometry and electronic structure of defective BlueP monolayers upon absorption of molecules. The equilibrium distance of the three molecules with respect to the pristine, $\mathrm{MV}$-, $\mathrm{Si}$ - and $\mathrm{S}-\mathrm{BlueP}$ monolayer is summarized in Table 1. The much reduced equilibrium distance in the defective BlueP monolayers indicates the stronger binding of these molecules. The top and side views of the acetone, ethanol, and propanal molecule absorbed on the MV-, Si- and S-BlueP monolayer are presented in Figure $5(\mathrm{a}-\mathrm{c})$, Figure $6(\mathrm{a}-\mathrm{c})$ and Figure $7(\mathrm{a}-\mathrm{c})$. These geometrical configurations are used for the electronic structure calculations.

Regarding the MV-BlueP monolayer, a major change in electronic structure near the Fermi level is observed upon the absorption of the three molecules, as evidenced by the DOS (Figure 5 (d-f)) and band structure plots (Figure S5). Before absorption, a couple of defect bands are found crossing the Fermi level, while upon absorption of the three molecules, such crossed bands split and shift away from the Fermi level (Figure 5 (d-f) and Figure S5). Such change leads to a change in carrier concentration, which may result in a visible change in electrical conductivity that can be detected for sensing of these three molecules. 

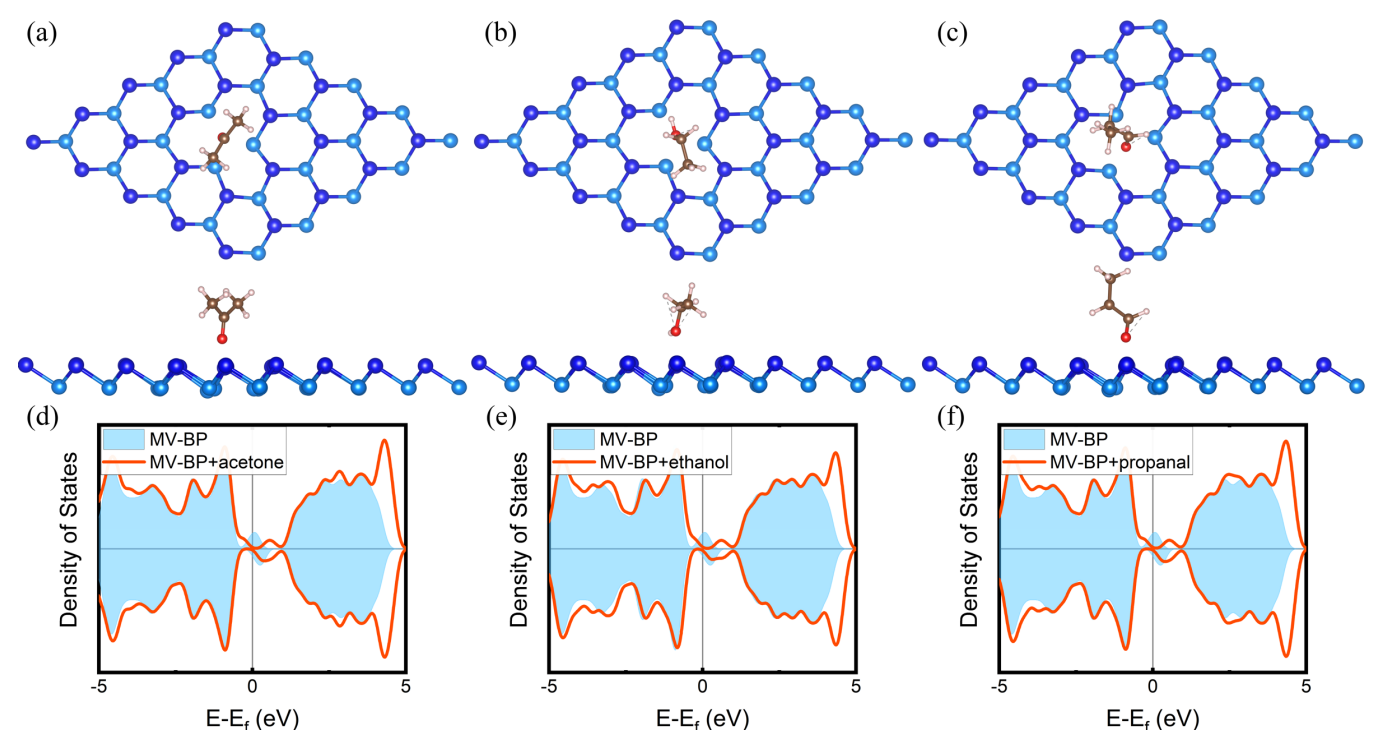

Figure 5. Top and side view of the most stable configurations of acetone (a), ethanol (b), and propanal (c) on MV-BP, and their respective density of states (DOS) plots (d-f). Atomic color scheme: $\mathrm{P}$ atoms in upper layer of BlueP, dark blue; $\mathrm{P}$ atoms in lower layer of BlueP, light blue; $\mathrm{H}$, pink; C, brown; and $\mathrm{O}$, red.

As regards the other electron-deficient BlueP monolayer, Si-BlueP shows one pair of spin-up and spin-down defect bands near the Fermi level, while they do not cross the Fermi level (Figure S6 (a)). Marginal change in DOS and band structure is found upon the absorption of ethanol, while the spin-down band is no longer found near the Fermi level upon the absorption of acetone and propanal (Figure 6 (d-f) and Figure S6). The vanishing of the spin-down middle gap band may also lead to some change in carrier concentration and thereby electrical conductivity, but such change should be much smaller than that of the MV-BlueP case.

For S-BlueP monolayer, the S substitution provides one more $p$ electron, which shifts the Fermi level towards the conduction band. The pair of spin-up and spin-down bands brought by this additional electron appears near the conduction band and crosses the Fermi level (Figure S7 (a)). Upon absorption the three molecules, the Fermi level is shifted towards the middle of the band gap, and the defect bands no long cross the Fermi level. Such major change in electronic structure should render S-BlueP monolayer as a suitable candidate for the sensing of these three molecules. 


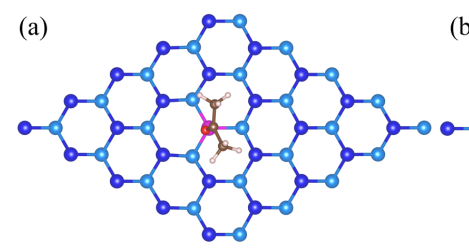

(b)
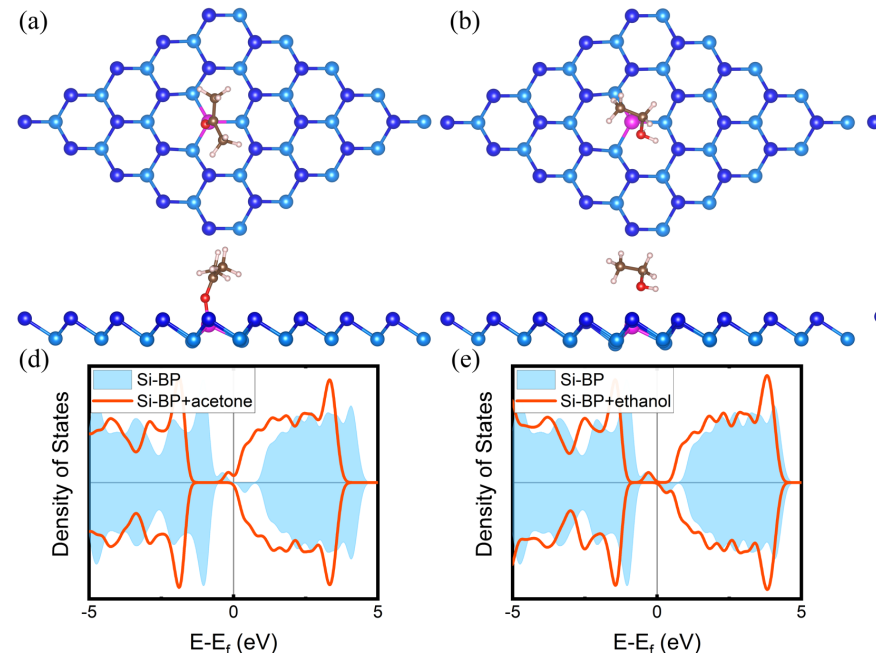

(c)

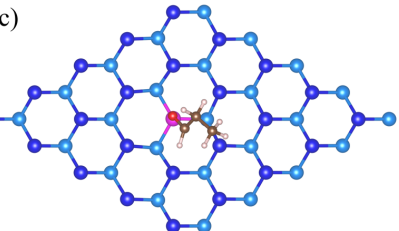

d.t.
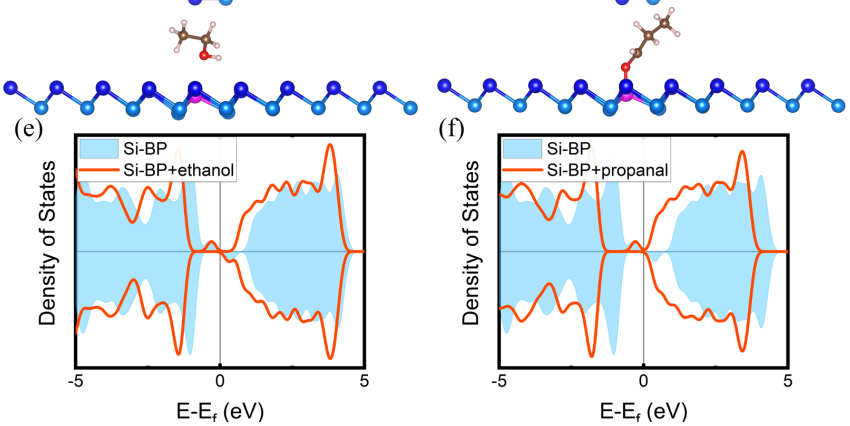

Figure 6. Top and side views of the most stable configurations of acetone (a), ethanol (b), and propanal (c) on Si-BlueP, and their respective density of states (DOS) plots (d-f). Atomic color scheme: $\mathrm{P}$ atoms in upper layer of BlueP, dark blue; $\mathrm{P}$ atoms in lower layer of BlueP, light blue; $\mathrm{H}$, pink; $\mathrm{C}$, brown; and $\mathrm{O}$, red; and $\mathrm{Si}$, purple.

(a)
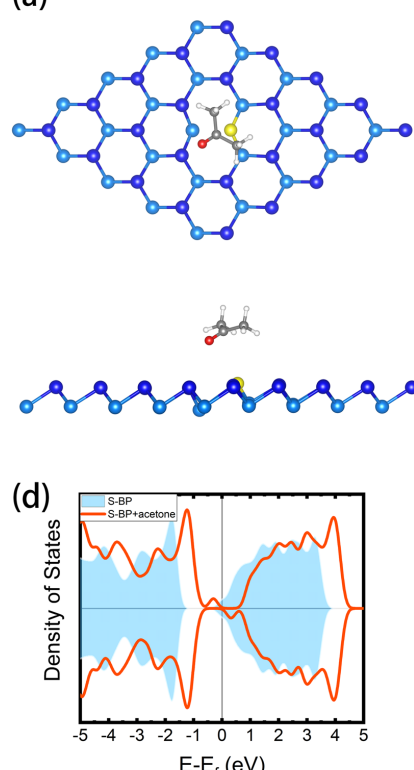

(b)

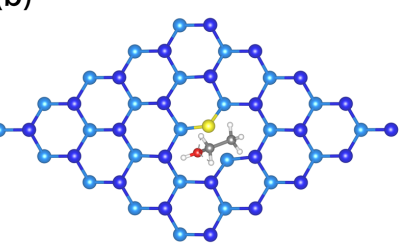

(c)
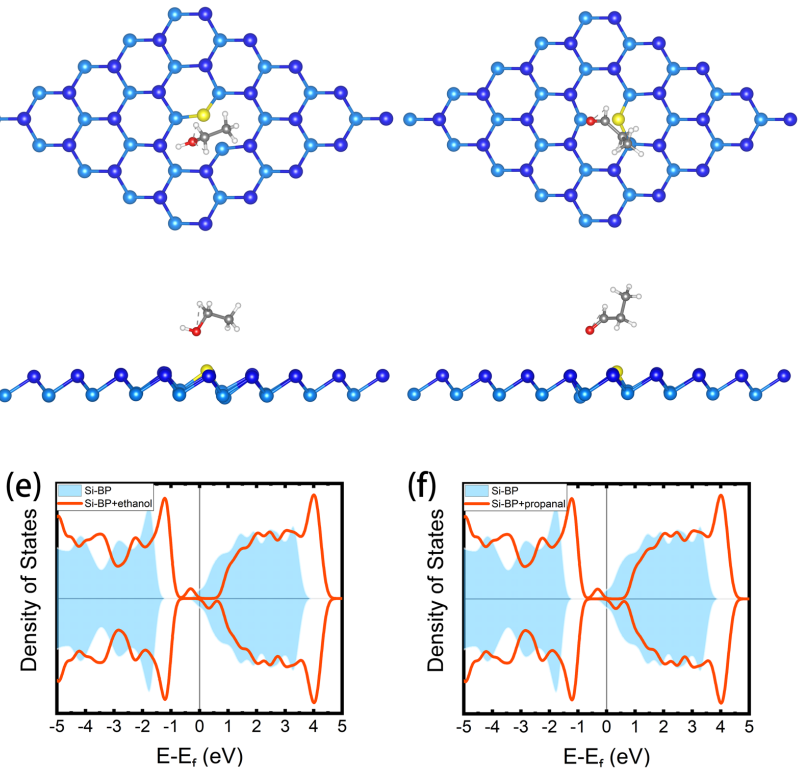

Figure 7 Top and side views of the most stable configurations of acetone (a), ethanol (b), and propanal (c) on S-BlueP, and their respective density of states (DOS) plots (d-f). Atomic color scheme: $\mathrm{P}$ atoms in upper layer of BlueP, dark blue; $\mathrm{P}$ atoms in lower layer of BlueP, light blue; $\mathrm{H}$, pink; C, brown; and $\mathrm{O}$, red; and S, yellow. 


\section{Conclusions}

Spin-polarized DFT calculations were carried out to investigate the structural, electronic, and sensing characteristics of pristine, MV- and Si/S-doped blue phosphorene monolayer with and without the absorption of volatile organic molecules, including acetone, ethanol, and propanal. Our energy analysis reveals favorable absorption tendency of the three molecules by pristine BlueP, however, the marginal change in electronic structure rules out pristine BlueP as potential gas nanosensors. The binding energy of between the three molecules and BlueP monolayer significantly increases upon the formation of vacancies and element substitution. The relatively low formation energy of mono-vacancy, $\mathrm{Si}$ and $\mathrm{S}$ substitution (within a 55 supercell of BlueP monolayer) makes these defective BlueP monolayers potentially feasible for synthesis. Moreover, major changes in electronic structure before and after the absorption of the three molecules are found in the MV- and S-BlueP monolayer, which shall lead to detectable changes in electrical conductivity, suggesting these two defective BlueP monolayers to be potentially good gas nanosensors.

\section{Acknowledgements}

This research was undertaken with the assistance of computational resources at the HPC platform of Xi'an Jiaotong University and the Linux cluster of the Karton group at the University of Western Australia. We gratefully acknowledge the provision of an Australian Research Council (ARC) Future Fellowship (to A.K.; Project No. FT170100373). The support of the International Joint Laboratory for Micro/Nano Manufacturing and Measurement Technologies (IJL-MMMT) of Xi'an Jiaotong University is also acknowledged. 


\section{References}

1. Guo, H.; Lee, S. C.; Chan, L. Y.; Li, W. M., Risk assessment of exposure to volatile organic compounds in different indoor environments. Environmental Research 2004, 94, 57.

2. Salthammer, T., Very volatile organic compounds: an understudied class of indoor air pollutants. Indoor Air 2016, 26, 25.

3. Wang, S.; Ang, H. M.; Tade, M. O., Volatile organic compounds in indoor environment and photocatalytic oxidation: state of the art. Environ Int 2007, 33, 694.

4. Molhave, L., Volatile Organic Compounds, Indoor Air Quality and Health. Indoor Air $1991,1,357$.

5. Bartzis, J.; Wolkoff, P.; Stranger, M.; Efthimiou, G.; Tolis, E. I.; Maes, F.; Norgaard, A. W.; Ventura, G.; Kalimeri, K. K.; Goelen, E.; Fernandes, O., On organic emissions testing from indoor consumer products' use. J Hazard Mater 2015, 285, 37.

6. Mirzaei, A.; Leonardi, S. G.; Neri, G., Detection of hazardous volatile organic compounds (VOCs) by metal oxide nanostructures-based gas sensors: A review. Ceramics International 2016, 42, 15119.

7. Dral, A. P.; ten Elshof, J. E., 2D metal oxide nanoflakes for sensing applications: Review and perspective. Sensors and Actuators B-Chemical 2018, 272, 369.

8. Gupta Chatterjee, S.; Chatterjee, S.; Ray, A. K.; Chakraborty, A. K., Graphene-metal oxide nanohybrids for toxic gas sensor: A review. Sensors and Actuators B: Chemical 2015, 221,1170 .

9. Zhang, Z.; Zou, X.; Xu, L.; Liao, L.; Liu, W.; Ho, J.; Xiao, X.; Jiang, C.; Li, J., Hydrogen gas sensor based on metal oxide nanoparticles decorated graphene transistor. Nanoscale 2015, 7, 10078.

10. V. E. Bochenkov, G. B. S., Sensitivity, Selectivity, and Stability of Gas-Sensitive MetalOxide Nanostructures. Metal Oxide Nanostructres and Their Applications 2010, 3.

11. Capone, S.; Forleo, A.; Francioso, L.; Rella, R.; Siciliano, P.; Spadavecchia, J.; Presicce, D. S.; Taurino, A. M., Solid state gas sensors: State of the art and future activities. Journal of Optoelectronics and Advanced Materials 2003, 5, 1335.

12. Schedin, F.; Geim, A. K.; Morozov, S. V.; Hill, E. W.; Blake, P.; Katsnelson, M. I.; Novoselov, K. S., Detection of individual gas molecules adsorbed on graphene. Nat Mater 2007, 6, 652 .

13. Karmaoui, M.; Leonardi, S. G.; Latino, M.; Tobaldi, D. M.; Donato, N.; Pullar, R. C.; Seabra, M. P.; Labrincha, J. A.; Neri, G., Pt-decorated $\operatorname{In}_{2} \mathrm{O}_{3}$ nanoparticles and their ability as a highly sensitive $(<10 \mathrm{ppb})$ acetone sensor for biomedical applications. Sensors and Actuators B: Chemical 2016, 230, 697. 
14. Farokh Niaei, A. H.; Hussain, T.; Hankel, M.; Searles, D. J., Hydrogenated defective graphene as an anode material for sodium and calcium ion batteries: A density functional theory study. Carbon 2018, 136, 73.

15. Hussain, T.; De Sarkar, A.; Ahuja, R., Functionalization of hydrogenated graphene by polylithiated species for efficient hydrogen storage. International Journal of Hydrogen Energy 2014, 39, 2560.

16. Zhou, X.; Zhang, Q.; Gan, L.; Li, H.; Xiong, J.; Zhai, T., Booming Development of Group IV-VI Semiconductors: Fresh Blood of 2D Family. Adv Sci (Weinh) 2016, 3, 1600177.

17. Manzeli, S.; Ovchinnikov, D.; Pasquier, D.; Yazyev, O. V.; Kis, A., 2D transition metal dichalcogenides. Nature Reviews Materials 2017, 2.

18. Schaibley, J. R.; Yu, H.; Clark, G.; Rivera, P.; Ross, J. S.; Seyler, K. L.; Yao, W.; Xu, X., Valleytronics in 2D materials. Nature Reviews Materials 2016, 1.

19. Wang, Q. H.; Kalantar-Zadeh, K.; Kis, A.; Coleman, J. N.; Strano, M. S., Electronics and optoelectronics of two-dimensional transition metal dichalcogenides. Nat Nanotechnol 2012, 7, 699.

20. Wang, J.; Ronneberger, I.; Zhou, L.; Lu, L.; Deringer, V. L.; Zhang, B.; Tian, L.; Du, H.; Jia, C.; Qian, X.; Wuttig, M.; Mazzarello, R.; Zhang, W., Unconventional twodimensional germanium dichalcogenides. Nanoscale 2018, 10, 7363.

21. Zhou, J.; Sun, Q.; Wang, Q.; Jena, P., High-temperature superconductivity in heavily Nor B-doped graphene. Physical Review B 2015, 92.

22. Huang, B.; Li, Z.; Liu, Z.; Zhou, G.; Hao, S.; Wu, J.; Gu, B.-L.; Duan, W., Adsorption of Gas Molecules on Graphene Nanoribbons and Its Implication for Nanoscale Molecule Sensor. The Journal of Physical Chemistry C 2008, 112, 13442.

23. Zhang, Y. H.; Chen, Y. B.; Zhou, K. G.; Liu, C. H.; Zeng, J.; Zhang, H. L.; Peng, Y., Improving gas sensing properties of graphene by introducing dopants and defects: a firstprinciples study. Nanotechnology 2009, 20, 185504.

24. Tian, X.-Q.; Liu, L.; Wang, X.-R.; Wei, Y.-D.; Gu, J.; Du, Y.; Yakobson, B. I., Engineering of the interactions of volatile organic compounds with MoS2. Journal of Materials Chemistry C 2017, 5, 1463.

25. Sarkar, D.; Xie, X.; Kang, J.; Zhang, H.; Liu, W.; Navarrete, J.; Moskovits, M.; Banerjee, K., Functionalization of transition metal dichalcogenides with metallic nanoparticles: implications for doping and gas-sensing. Nano Lett 2015, 15, 2852.

26. Li, L.; Yu, Y.; Ye, G. J.; Ge, Q.; Ou, X.; Wu, H.; Feng, D.; Chen, X. H.; Zhang, Y., Black phosphorus field-effect transistors. Nat Nanotechnol 2014, 9, 372.

27. Kou, L.; Frauenheim, T.; Chen, C., Phosphorene as a Superior Gas Sensor: Selective Adsorption and Distinct I-V Response. J Phys Chem Lett 2014, 5, 2675.

28. Abbas, A. N.; Liu, B.; Chen, L.; Ma, Y.; Cong, S.; Aroonyadet, N.; Kopf, M.; Nilges, T.; 
Zhou, C., Black phosphorus gas sensors. ACS Nano 2015, 9, 5618.

29. Yang, Q.; Meng, R.-S.; Jiang, J.-K.; Liang, Q.-H.; Tan, C.-J.; Cai, M.; Sun, X.; Yang, D.G.; Ren, T.-L.; Chen, X.-P., First-Principles Study of Sulfur Dioxide Sensor Based on Phosphorenes. IEEE Electron Device Letters 2016, 37, 660.

30. Kaewmaraya, T.; Ngamwongwan, L.; Moontragoon, P.; Karton, A.; Hussain, T., Drastic Improvement in Gas-Sensing Characteristics of Phosphorene Nanosheets under Vacancy Defects and Elemental Functionalization. The Journal of Physical Chemistry C 2018.

31. Yang, A.-J.; Wang, D.-W.; Wang, X.-H.; Chu, J.-F.; Lv, P.-L.; Liu, Y.; Rong, M.-Z., Phosphorene: A Promising Candidate for Highly Sensitive and Selective SF6 Decomposition Gas Sensors. IEEE Electron Device Letters 2017, 38, 963.

32. Liu, H.; Neal, A. T.; Zhu, Z.; Luo, Z.; Xu, X.; Tomanek, D.; Ye, P. D., Phosphorene: an unexplored 2D semiconductor with a high hole mobility. ACS Nano 2014, 8, 4033.

33. Mahabal, M. S.; Deshpande, M. D.; Hussain, T.; Ahuja, R., Sensing Characteristics of Phosphorene Monolayers toward PH3 and AsH3 Gases upon the Introduction of Vacancy Defects. The Journal of Physical Chemistry C 2016, 120, 20428.

34. Balendhran, S.; Walia, S.; Nili, H.; Sriram, S.; Bhaskaran, M., Elemental analogues of graphene: silicene, germanene, stanene, and phosphorene. Small 2015, 11, 640.

35. Kou, L.; Chen, C.; Smith, S. C., Phosphorene: Fabrication, properties, and applications. Journal of Physical Chemistry Letters 2015, 6, 2794.

36. Zhu, Z.; Tomanek, D., Semiconducting layered blue phosphorus: a computational study. Phys Rev Lett 2014, 112, 176802.

37. Zhang, J. L.; Zhao, S.; Han, C.; Wang, Z.; Zhong, S.; Sun, S.; Guo, R.; Zhou, X.; Gu, C. D.; Yuan, K. D.; Li, Z.; Chen, W., Epitaxial Growth of Single Layer Blue Phosphorus: A New Phase of Two-Dimensional Phosphorus. Nano Lett 2016, 16, 4903.

38. Kresse, G.; Furthmüller, J., Efficient iterative schemes forab initiototal-energy calculations using a plane-wave basis set. Physical Review B 1996, 54, 11169.

39. Blochl, P. E., Projector Augmented-Wave Method. Physical Review B 1994, 50, 17953.

40. Perdew, J. P.; Burke, K.; Ernzerhof, M., Generalized Gradient Approximation Made Simple. Phys Rev Lett 1996, 77, 3865.

41. Grimme, S., Semiempirical GGA-type density functional constructed with a long-range dispersion correction. J Comput Chem 2006, 27, 1787.

42. Paier, J.; Marsman, M.; Hummer, K.; Kresse, G.; Gerber, I. C.; Angyan, J. G., Screened hybrid density functionals applied to solids. J Chem Phys 2006, 124, 154709. 